Check for updates

Cite this: RSC Adv., 2019, 9, 22966

Received 28th May 2019

Accepted 15th July 2019

DOI: 10.1039/c9ra04026a

rsc.li/rsc-advances

\title{
Hybrid cellulose nanocrystal/alginate/gelatin scaffold with improved mechanical properties and guided wound healing
}

\author{
Yue Shan, Chaoyue Li, Yongzhi Wu, Qiwen Li and Jinfeng Liao (iD)*
}

\begin{abstract}
Nature derived biopolymers such as polysaccharides and collagen have attracted considerable attention in biomedical applications. Despite excellent biocompatibility and bioactivity, their poor mechanical properties could not meet the requirement for skin regeneration. In this study, cellulose nanocrystal (CNC) was incorporated into the calcium cross-linked sodium alginate/gelatin (SA/Ge) scaffold to reinforce its physicochemical properties. A novel sodium alginate/gelatin/cellulose nanocrystal (SA/Ge/ $\mathrm{CNC}$ ) scaffold was successfully prepared through electrostatic interaction of sodium alginate and gelatin, ionic cross-linking of calcium ions with sodium alginate, and incorporation of CNC. Afterwards, the SA/ Ge and SA/Ge/CNC scaffolds were fully characterized and compared with scanning electron microscopy images, swelling behaviors, tensile strengths and contact angles. The involvement of CNC produces a hybrid SA/Ge/CNC scaffold with desired porous network, moderate swelling behavior, and superior mechanical strength (from $18 \mathrm{MPa}$ to $45 \mathrm{MPa}$ ). Furthermore, in vitro cytotoxicity and cell growth assay using mouse embryonic fibroblast cells validated that SA/Ge/CNC scaffold was non-toxic and can prompt cell adhesion and proliferation. The in vivo skin regeneration experiments using the SA/Ge/CNC scaffold group showed an improved skin wound healing process with accelerated re-epithelialization, increased collagen deposition and faster extracellular matrix remodeling. Overall, the results suggested that the SA/Ge/CNC hybrid scaffold with enhanced mechanical performance and wound healing efficacy was a promising biomaterial for skin defect regeneration.
\end{abstract}

\section{Introduction}

Skin is the largest and one of the most important organs of humans, acting as a protective blockage to the invasion of harmful microorganisms and damage from certain environmental conditions. ${ }^{1,2}$ Dermal tissue defects following burns, surgical excision, skin trauma and chronic ulcers remain a challenging clinical problem and cause a substantial cost burden on the public. ${ }^{3,4}$ The traditional treatments for skin repair include implantation of autologous, allogeneic and exogenous skin grafts, which are usually constricted by the lack of donor sources, costly medical expenses and undesirable immunological response. ${ }^{5,6}$ Therefore, it is urgently desirable to develop an ideal substitute which is structurally, functionally, and mechanically similar to healthy skin. ${ }^{7}$ The recovery of injured skin tissue is a highly ordered biological process, which involves hemostasis, inflammation reaction, formation of granulation tissue, re-epithelialization, and matrix remodeling. ${ }^{8,9}$ With better understanding of the wound repair and management process, numerous biomaterials have been

State Key Laboratory of Oral Diseases, National Clinical Research Centre for Oral Diseases, West China Hospital of Stomatology, Sichuan University, Chengdu, 610041, China. E-mail: liaojinfeng.762@163.com created to mimic the natural environment for skin regeneration, and accelerate cell proliferation and induce extracellular matrix (ECM) reproduction. ${ }^{\mathbf{1 0 , 1 1}}$

To date, a number of naturally derived polymers, including polysaccharides and proteins have been widely utilized in the field of tissue engineering scaffolds, due to their excellent hydrophilicity, biodegradability and biocompatibility. ${ }^{\mathbf{1 2 - 1 4}}$ In particular, sodium alginate, a seaweed-derived polysaccharides, is preferred for its gelling property, excellent biocompatibility, and source-abundance. ${ }^{15-17}$ Besides, alginate is composed of consecutive and alternately arranged $\alpha$-L-guluronic and $\beta$-Dmannuronic blocks and is able to cross-link with divalent metal ions (e.g. $\left.\mathrm{Ca}^{2+}, \mathrm{Zn}^{2+}\right) \cdot{ }^{18,19}$ Collagen is an important component of skin and provides resistance to connective tissues. Gelatin is a hydrolyzed product from collagen with desired features of prompting hemostasis and guiding cell adherence, proliferation and differentiation. ${ }^{15,20}$ Combining two or more different polymers by cross-linkers has been reported to be an effective method to create a new scaffold, which can get rid of their disadvantages and integrate their advantages. ${ }^{21,22}$ For example, the composite materials blending alginate with gelatin together have gained much attention because of superior physicochemical and biological properties. ${ }^{23,24}$ That hybrid alginate/ gelatin materials get over the respective drawbacks of 
constituents, such as the rapid degradation rate, high swelling degree of gelatin, and weak mechanical properties of alginate. ${ }^{25}$ Nevertheless, the composite materials still existed some limitations like inadequate mechanical strength and loose network structure. $^{26}$

To overcome these deficiencies, we integrated cellulose nanocrystals (CNC) into the composite scaffolds as a functional additive to improve their physical, chemical and biological performances. CNC is a kind of unique nanomaterial, which derived from natural cellulose fibers. ${ }^{27}$ It has excellent properties of high aspect ratio, low toxicity, excellent mechanical performance and good biocompatibility. One major application of CNC is acting as reinforcing agent in composites. It is well known that the high mechanical strength of CNC ascribed to its highly crystalline structure and the existence of intra- and intermolecular hydrogen bonds. The incorporation of CNC can form effective interfacial interaction between the reinforcement material and matrix. Furthermore, the large surface area and abundant surface hydroxyl groups of CNC provide the possibility of chemical modifications and drug delivery. Due to its outstanding characteristics, CNC has been considered as an ideal reinforcing component for polymer matrix integration. ${ }^{21,28,29}$ The combination of CNC with sodium alginate and gelatin is expected to synthesize a nanocomposite with improved mechanical and biological properties.

To date, most skin substitutes and tissue engineered scaffolds are still in the research and development stages. The current commercialized products such as Dermagraft, Apligraf, MatriDerm, PriMatrix and AlloDerm should be viewed as "smart dressings", rather than true skin equivalents. They are cellularized, decellularized or bioengineered matrices that maintain and support a healing environment through facilitating cell adhesion and proliferation, as well as skin appendages regeneration. ${ }^{30}$ In this study, a novel hybrid scaffold comprised of sodium alginate, gelatin and $\mathrm{CNC}$ was prepared by using the solvent casting method. Compared with the commercial products, the $\mathrm{SA} / \mathrm{Ge} / \mathrm{CNC}$ scaffold reconstructs and remodels wounds in a similar way. Moreover, the SA/Ge/CNC scaffold is inexpensive, renewable, biocompatible, and easy to prepare in a large scale.

As shown in Scheme 1A, the synthesis of SA/Ge/CNC scaffold involved electrostatic interaction of gelatin and alginate, incorporation of cellulose nanocrystals and crosslinking of alginate with calcium ions. The appropriate sodium alginate/ gelatin ratio and CNC concentration were systemically investigated and chosen. All the scaffolds were characterized in terms of their structures, swelling behaviors, mechanical properties and contact angles. Additionally, mouse embryonic fibroblast cells (NIH 3T3) were used to assess the in vitro cytocompatibility of scaffolds. Finally, in vivo wound repair experiments on full-thickness excision wound models of SD rats were further implemented to evaluate their biological application potential through both macroscopic and microscopic observation (Scheme 1B). The results demonstrated that SA/Ge/CNC scaffold can be considered as a potential substitute for skin tissue engineering.

\section{Materials and methods}

\subsection{Materials}

Sodium alginate (from brown algae, molecular weight of 20-40 $\mathrm{kDa}$ ) and gelatin (Type $\mathrm{B}$, from porcine skin) were purchased from Sigma-Aldrich (USA). Cellulose nanocrystals (CNC, extracted from cotton pulp, prepared by sulfuric acid hydrolysis, length between 150-200 $\mathrm{nm}$, diameter 4-7 nm; solid content about 1.2 wt\% in suspension) was obtained from Haojia Chemicals, Tianjin, China. Anhydrous $\mathrm{CaCl}_{2}$, chloral hydrate $(10 \%)$ and phosphate buffer saline (PBS, $\mathrm{pH}=7.4)$ were acquired from Kelong Chemicals, Chengdu, China. 3-(4,5Dimethylthiazol-2-yl)-2,5-diphenyltetrazolium bromide (MTT) and dimethyl sulfoxide (DMSO) were purchased from Shanghai Aladdin Bio-Chem Technology Co., Ltd. Dulbecco's Modified Eagle's Medium (DMEM), fetal bovine serum (FBS), penicillin and all other culture media and reagents were purchased from Gibco $^{\mathrm{TM}}$ (United States). All the reagents were of laboratory reagent grade.

For the skin regeneration study, 12 male rats weighing 200$220 \mathrm{~g}$ were obtained from Chengdu Dashuo Experimental Animal Co., Ltd. All the surgical procedures on rats were approved by the animal care and use committee of State Key Laboratory of Oral Diseases, Sichuan University. During the experiment, all the animals were kept in individual cages and were fed with a commercial rat food and water ad libitum.

\subsection{Methods}

2.2.1 Preparation of sodium alginate/gelatin scaffold. The sodium alginate was dissolved in distilled water (3 wt\%) under continuous stirring for $18 \mathrm{~h}$ at room temperature until a homogeneous solution was obtained. The aqueous solution of gelatin ( $3 \mathrm{wt} \%$ ) was prepared by dispersing gelatin powder into distilled water at $60{ }^{\circ} \mathrm{C}$ under constant stirring for $2 \mathrm{~h}$ to ensure complete dissolution. Subsequently, the two biopolymer solutions were vigorously mixed together at different ratios and stirred at room temperature for $24 \mathrm{~h}$. After sonication for $1 \mathrm{~h}$ to get rid of remaining air bubbles, the resulting SA/Ge blend solution with designed ratios were poured into Petri dishes $(d=$ $90 \mathrm{~mm}$ ). To dry the solution and get the dried films, the Petri dishes were placed into a normal oven at $45{ }^{\circ} \mathrm{C}$ for 2 days. Afterwards, $15 \mathrm{ml} \mathrm{CaCl}_{2}$ aqueous solution (2 wt\%) was added into each Petri dish for $30 \mathrm{~min}$ to achieve cross-linking of sodium alginate with $\mathrm{Ca}^{2+}$. Then the scaffolds were washed with distilled water for three times to remove the extra free reagents. Ultimately, the prepared scaffolds were put into the oven again for drying until the weight didn't change any more. The detailed content and varying volume ratios of alginate and gelatin are listed in Table 1.

2.2.2 Preparation of sodium alginate/gelatin/CNC scaffold. To introduce $\mathrm{CNC}$ into $\mathrm{SA} / \mathrm{Ge}$ scaffold, the original $\mathrm{CNC}$ aqueous solution (1.2 wt $\%$ ) was diluted to yield the final concentration of $0.1,0.5,1 \mathrm{wt} \%$, respectively. Then the CNC solutions of various concentrations were added into the SA/Ge mixture. Equal volume of distilled water was added to serve as a control sample. The SA/Ge/CNC composites with different 

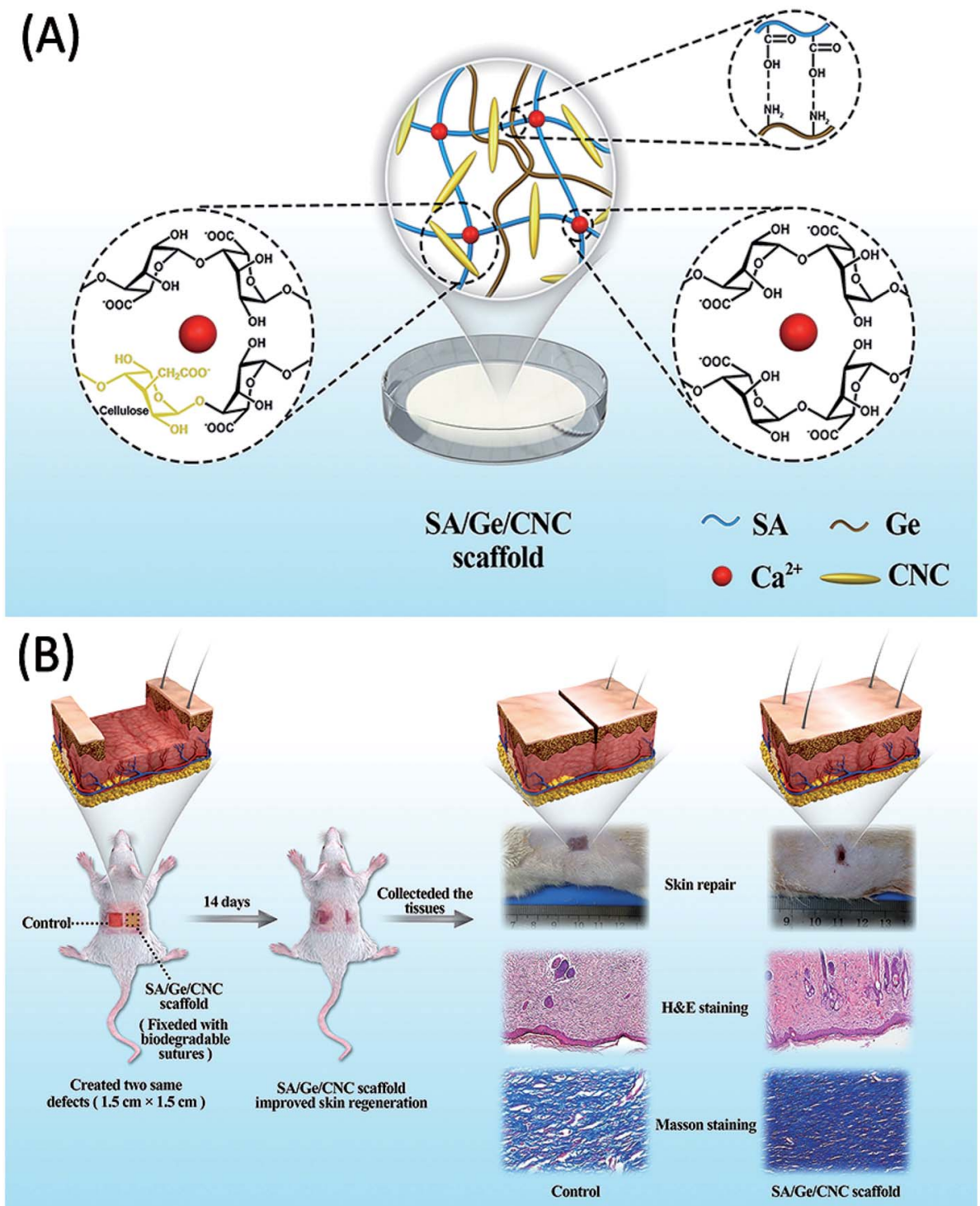

Scheme 1 (A) The illustration of the SA/Ge/CNC scaffold. (B) The skin regeneration improved by the SA/Ge/CNC scaffold in SD rats.

Table 1 The content and different ratios of alginate, gelatin and CNC for the synthesis of SA/Ge and SA/Ge/CNC scaffolds

\begin{tabular}{lllll}
\hline Sample & $\begin{array}{l}3 \mathrm{wt} \% \\
\text { Alginate }(\mathrm{ml})\end{array}$ & $\begin{array}{l}3 \mathrm{wt} \% \\
\text { Gelatin }(\mathrm{ml})\end{array}$ & $\begin{array}{l}1 \mathrm{wt} \% \\
\mathrm{CNC}(\mathrm{ml})\end{array}$ & $\begin{array}{l}\mathrm{H}_{2} \mathrm{O} \\
(\mathrm{ml})\end{array}$ \\
\hline $\mathrm{SA} / \mathrm{Ge}(1: 2)$ & 30 & 60 & 0 & 0 \\
$\mathrm{SA} / \mathrm{Ge}(1: 1)$ & 45 & 45 & 0 & 0 \\
$\mathrm{SA} / \mathrm{Ge}(2: 1)$ & 60 & 30 & 0 & 0 \\
$\mathrm{SA} / \mathrm{Ge}(4: 1)$ & 72 & 18 & 0 & 0 \\
$\mathrm{SA} / \mathrm{Ge} / \mathrm{CNC}(0)$ & 40 & 20 & 0 & 5 \\
$\mathrm{SA} / \mathrm{Ge} / \mathrm{CNC}(0.1 \%)$ & 40 & 20 & 0.5 & 4.5 \\
$\mathrm{SA} / \mathrm{Ge} / \mathrm{CNC}(0.5 \%)$ & 40 & 20 & 2.5 & 2.5 \\
$\mathrm{SA} / \mathrm{Ge} / \mathrm{CNC}(1 \%)$ & 40 & 20 & 5 & 0 \\
& & & &
\end{tabular}

ratios (Table 1) were stirred overnight to obtain the final homogeneous dispersion. The casting and $\mathrm{CaCl}_{2}$ cross-linking procedures were similar to the preparation process of $\mathrm{SA} / \mathrm{Ge}$ scaffolds mentioned above.

\subsection{Characterization of scaffolds}

2.3.1 The morphological characterization of CNC and scaffolds. The aqueous solution of $\mathrm{CNC}$ was diluted to the concentration of $0.01 \mathrm{wt} \%$ and its morphology was studied by atomic force microscopy (AFM; Seiko Instruments Inc, Japan). For the cross-section morphology observation, the SA/Ge scaffolds and SA/Ge/CNC scaffolds were lyophilized and characterized using scanning electron microscopy (SEM; JSM-5900LV, JEOL, Tokyo, Japan) at an accelerating voltage of $15 \mathrm{kV}$. Meanwhile, a more accurate measurement of the average pore size was done with the application of software Smile-View. 100 pores were chosen randomly in each kind of scaffold from their SEM images to estimate the average pore size. Besides, in order to obtain the three-dimensional (3D) reconstruction and estimate the porosity of lyophilized scaffolds, micro-CT analyze was performed using an X-ray CT scanner (SKYSCAN 1172, German). 
2.3.2 Fourier transform infrared spectroscopy (FTIR). FTIR spectroscopic measurements were carried out for characterization of functional groups of SA/Ge scaffold and their interactions with CNC. The FTIR spectra of freeze-dried CNC, SA/Ge scaffold and SA/Ge/CNC scaffold were characterized using a Thermo Nicolet 6700 spectrometer.

2.3.3 Swelling behavior. The swelling degree of $\mathrm{SA} / \mathrm{Ge}$ scaffolds and SA/Ge/CNC scaffolds were measured by a general gravimetric method. First of all, the dried scaffolds of each group were cut into $1 \mathrm{~cm} \times 3 \mathrm{~cm}$ strips and their dry weight were documented. Then, all the samples were immersed in a PBS solution $(\mathrm{pH}=7.4)$ at room temperature. At predetermined time intervals $(t=5 \mathrm{~min}, 30 \mathrm{~min}, 1 \mathrm{~h}, 2 \mathrm{~h}, 6 \mathrm{~h}$, and $24 \mathrm{~h}$ ), the swollen samples were withdrawn and the excessive water on the sample surface was wiped off with filter paper. After weighing, the samples were placed into PBS solution again. The swelling degree was expressed as the following equation:

$$
\text { Swelling degree }(\%)=\left(W_{2}-W_{1}\right) / W_{1} \times 100 \%
$$

where $W_{2}$ and $W_{1}$ represent the weight of the swollen and dry samples, respectively.

2.3.4 Mechanical properties. The tensile behavior of scaffolds was determined using the mechanical testing instrument (INSTRON 5565, USA) at a tension mode. The scaffolds were sectioned into uniform rectangular strips $(1 \mathrm{~cm} \times 3 \mathrm{~cm})$ and the thickness was measured at five different locations by a digital micrometer caliper to calculate the average. All the samples were mounted on the mechanical testing instrument with a uniform gauge length of $15 \mathrm{~mm}$. Under a loading speed of 1 $\mathrm{mm} \min ^{-1}$, the scaffolds were stretched until reaching their breaking points. At least six replicates from each group were tested. The numbers of shifts and loading at rupture were recorded and the final tensile strength was calculated based on the formula:

$$
\sigma=\mathrm{Fb} / \mathrm{So}
$$

( $\sigma=$ tensile strength, $\mathrm{Fb}=$ the maximum force loading at the breaking points, So $=$ the cross-sectional area)

2.3.5 Contact angle. To investigate the influence of CNC on the hydrophilicity of scaffold, the $\mathrm{SA} / \mathrm{Ge} / \mathrm{CNC}$ scaffolds with various concentrations of $\mathrm{CNC}$ were detected by a contact angle measuring machine (Rame-Hart, Mountain Lakes, NJ, USA). Water droplet with a volume of $3 \mu \mathrm{l}$ was dropped on the scaffold to calculate the angle which the liquid-vapor interface made with the solid surface. The measurement was taken at four different points on each sample and the final contact angle was presented as the average value and standard derivation of four points.

\subsection{In vitro cell viability and cell morphology assay}

The in vitro cell viability of SA/Ge/CNC scaffold was measured by MTT assay. In this study, NIH 3T3 cells were selected. The cells were seeded in 96-well plates and incubated under a humidified atmosphere in the presence of $5 \% \mathrm{CO}_{2}$ at $37{ }^{\circ} \mathrm{C}$. After being sterilized with ethylene oxide for $8 \mathrm{~h}$, the scaffolds (100 mg) were then immersed in DMEM solution $(1 \mathrm{ml})$ for $24 \mathrm{~h}$ to extract the leachate. Subsequently, the original leachate $\left(100 \mathrm{mg} \mathrm{ml}^{-1}\right)$ were diluted to various concentrations $(0.78,1.56,3.13,6.25$, 12.5, 25, 50 and $\left.75 \mathrm{mg} \mathrm{ml}^{-1}, n=6\right)$. Then, $100 \mu$ leachate at each concentration was added into culture wells separately. The culture wells without leachate were set as control and wells with leachate were used as the experimental groups. After incubation for $1 \mathrm{~d}, 20 \mu \mathrm{l}$ of MTT solution $\left(5 \mathrm{mg} \mathrm{ml}^{-1}\right.$ ) was added into each well and further cultured at $37{ }^{\circ} \mathrm{C}$ for $4 \mathrm{~h}$. Afterwards, the medium was replaced by $150 \mu \mathrm{l}$ DMSO solution for each well to dissolve the formed formazan crystals. The ultra-violet (UV) absorbance at $570 \mathrm{~nm}$ was quantified using an ELISA reader (BioTek Instruments, Inc., Winooski, Vermont, USA).

For the cell morphology and adherence evaluation, NIH 3T3 cells were seeded onto $\mathrm{SA} / \mathrm{Ge} / \mathrm{CNC}$ scaffolds in humidified condition at $37{ }^{\circ} \mathrm{C}$ and $5 \% \mathrm{CO}_{2}$. After incubation for 3 days, the scaffolds were fixed with $2.5 \%$ glutaraldehyde solution for $2 \mathrm{~h}$ and then rinsed with $\mathrm{PBS}(\mathrm{pH}=7.4)$ for at least three times. Afterwards, the scaffolds were dehydrated twice in a series of graded ethanol. Finally, the samples were visualized by SEM and the images were photographed.

\subsection{Skin regeneration in SD rats}

Twelve healthy 8 week-old male SD rats (weighing 200-220 g) were randomly divided into two groups: (1) SA/Ge group, and (2) $\mathrm{SA} / \mathrm{Ge} / \mathrm{CNC}$ group; each group had 6 animals. All the rats were anaesthetized by an injection of $10 \%$ chloral hydrate $(2.5 \mathrm{ml}$ $\mathrm{kg}^{-1}$ ) and removed the dorsal hair with a shaving machine. After disinfecting with iodophor, two square full-thickness excision defects $(1.5 \mathrm{~cm} \times 1.5 \mathrm{~cm})$ were created symmetrically on the bilateral sides of each SD rat's back using surgical scissors (Scheme 1B). The SA/Ge scaffolds and SA/Ge/CNC scaffolds were covered on the right defects (anatomical position). At four corners of wounds, biodegradable sutures were applied for fixation. Wounds on the left side of both groups were set as a control, which were not treated with scaffolds. Finally, all the wounds on both sides were covered with dressings (3M Health Care, Germany) for protection. At 7 and 14 days after treatment, the macroscopic morphology of defects were observed and photographed at a stable distance. Then, rats were sacrificed to collect wound skin along with surrounding healthy tissues for further study ( 3 rats from each group on days 7 and 14 postwounding). The harvested tissue samples were fixed in $10 \%$ formaldehyde overnight. After a series of dehydration procedures with graded ethanol, the tissues were embedded in paraffin and sectioned into $5 \mu \mathrm{m}$ thick slices for hematoxylineosin (H\&E) and Masson's trichrome staining. The sections were observed by a microscope (Nikon ECLPSE, Japan) and the images were captured.

\subsection{Statistical analysis}

The statistical analysis was performed by SPSS 25.0 software (SPSS Inc., Chicago, IL, USA). The mean values and standard deviations were calculated for the pore size, swelling percentage, mechanical strength, contact angle, in vitro cell 
viability and in vivo skin defect regeneration research. $P$ values $<$ 0.05 indicates a statistically significant difference.

\section{Results and discussion}

\subsection{Selection of the optimum ratio of $\mathrm{SA} / \mathrm{Ge}$ in scaffold}

Before preparation of SA/Ge/CNC hybrid scaffold, the optimum ratio of SA and Ge in SA/Ge scaffold was investigated firstly. The scaffolds were measured various physical properties such as morphology, swelling behavior, and mechanical strength, etc. The pore size and distribution among scaffolds are highly related to cell adherence, proliferation, and nutrient exchange in matrix. ${ }^{20}$ Scaffold with porous structure renders fast efficiency of biomolecules transportation with environment and is an ideal material for the skin tissue engineering. ${ }^{31}$ Fig. $1 \mathrm{~A}$ shows the SEM photographs of SA/Ge scaffolds with different ratios at $\times 200$ magnification. The cross-section morphology of all scaffolds presented a porous and interconnected structure. The average pore dimension of scaffolds was displayed in Table 2. It was observed that as the ratios of $\mathrm{SA} / \mathrm{Ge}$ increased, pores in scaffolds became more homogeneous and the pore size appeared smaller. On the contrary, the increased proportion of Ge led to a more disordered and non-uniform composite network with larger pores. Additionally, the pore size of SA : Ge $=2: 1$ group and $\mathrm{SA}: \mathrm{Ge}=4: 1$ group showed no much significant difference in SEM images.

The swelling behavior of scaffolds is a crucial feature in terms of its potential for skin regeneration applications. An appropriate swelling degree can maintain the moist environment of wound and protect it from excessive dehydration. At the same time, the swelling ability influences the diffusion of nutrition and absorption of exudates. ${ }^{9}$ Thus, the swelling behavior of different SA/Ge scaffolds were tested. As shown in Fig. 1B, the four groups all indicated continuous enhancement of swelling degree and reached equilibrium after being immersed in PBS for approximately $6 \mathrm{~h}$. Compared with the
(A)
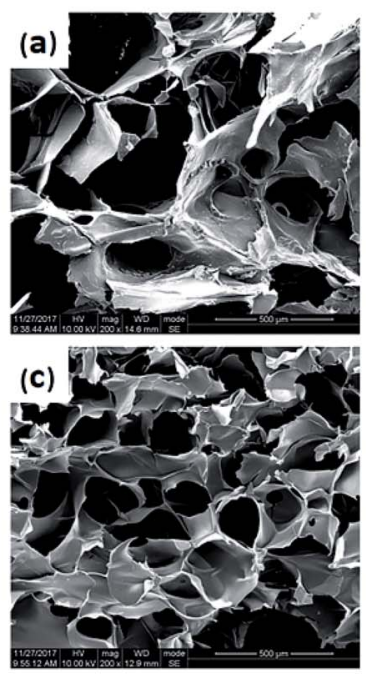

(C)

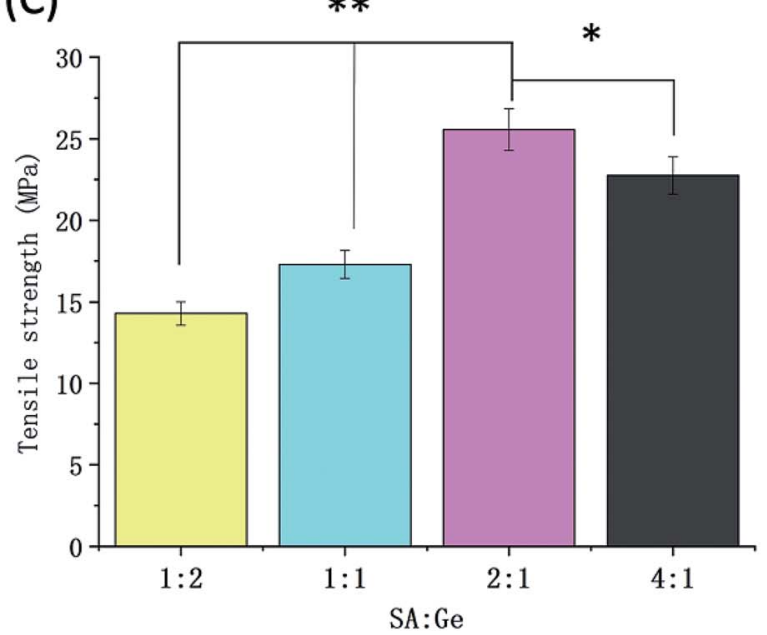

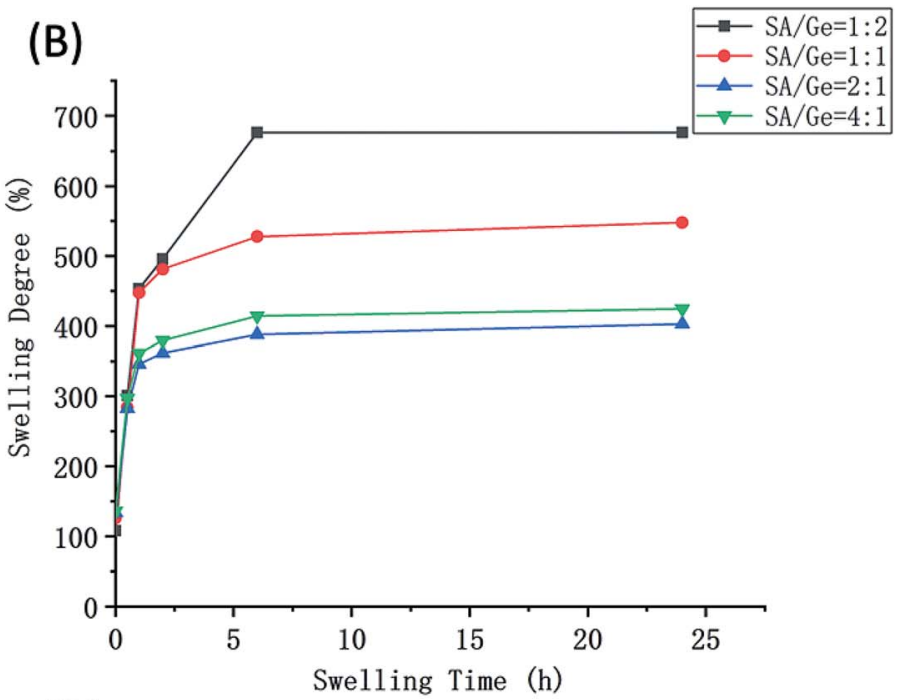

(D)

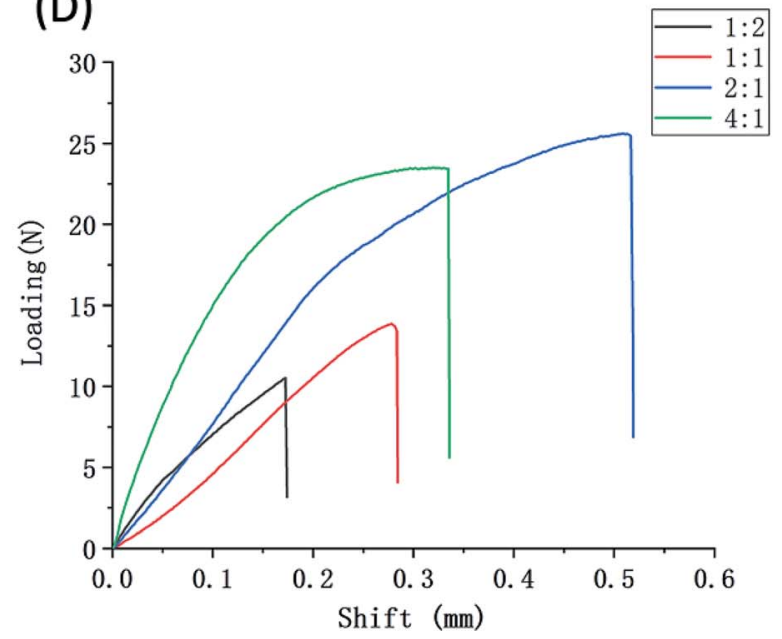

Fig. 1 Physical characteristics of SA/Ge scaffolds (A) SEM images of SA/Ge scaffolds with different SA/Ge ratios: (a) SA/Ge = 1:2, (b) SA/Ge = $1: 1$, (c) $S A / G e=2: 1$, (d) $S A / G e=4: 1$. The bar corresponds to $500 \mu \mathrm{m}$. (B) Swelling degree, (C) maximum tensile strength at breaking points, and (D) representative shift-loading curves of SA/Ge scaffolds in different ratios. * and ** denote significant difference at $p<0.05$ and $p<0.01$ levels, respectively. 
(A)

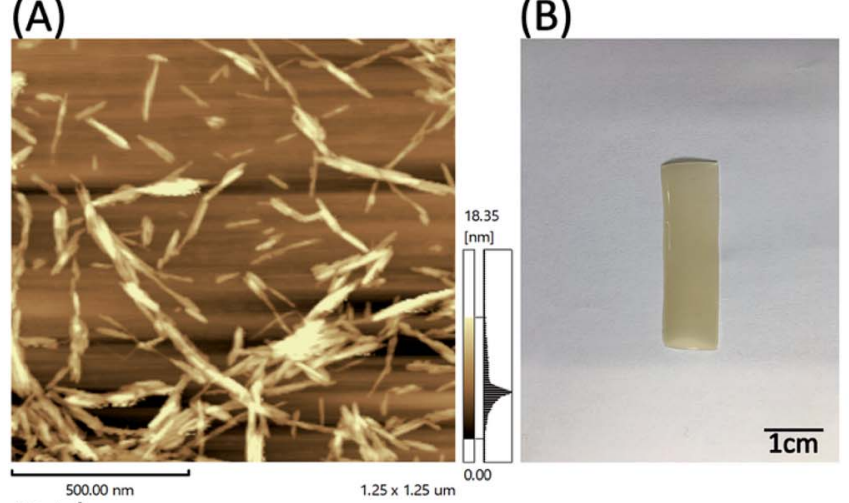

(C)

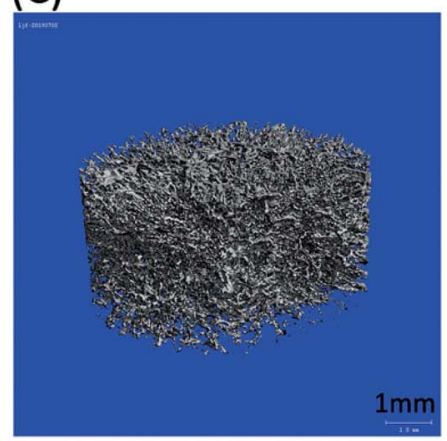

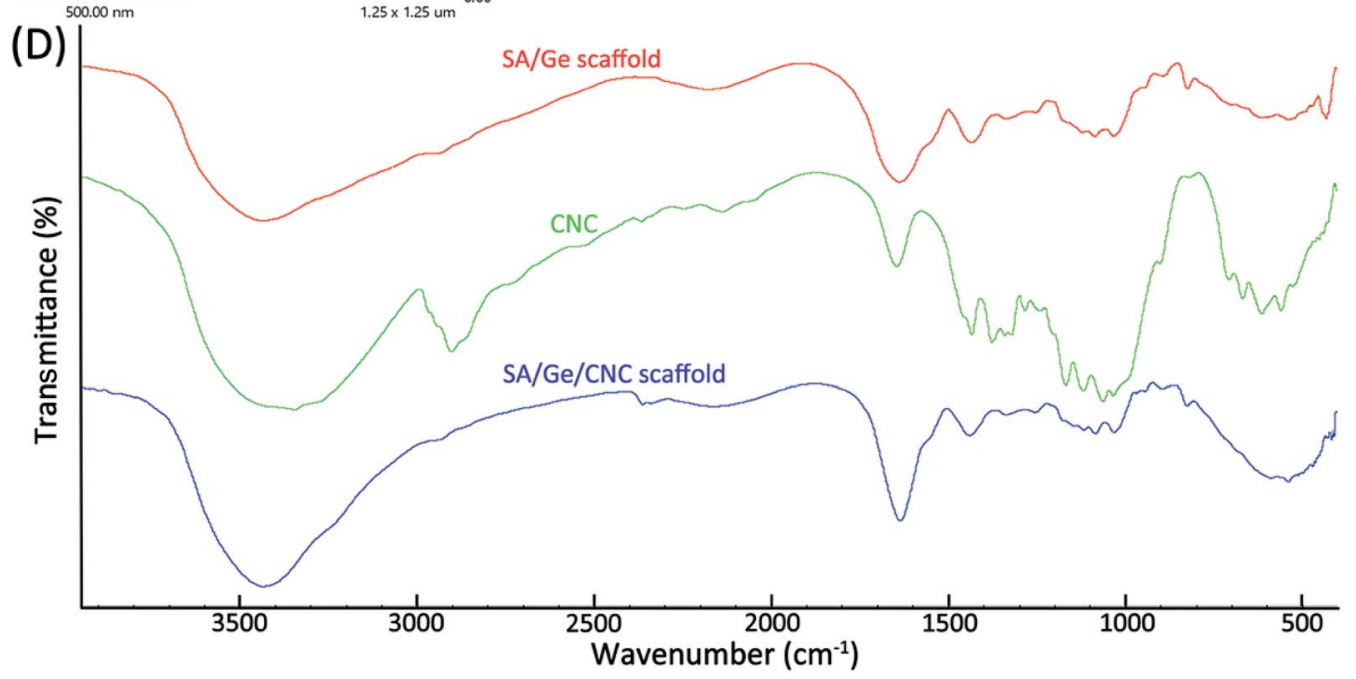

Fig. 2 (A) Atomic force microscopy image of $0.01 \mathrm{wt} \%$ CNC dispersed in aqueous solution. (B) Visual appearance of SA/Ge/CNC (SA : Ge = $2: 1$, $\mathrm{CNC}=0.5 \%$ ) scaffold (length $=3 \mathrm{~cm}$, width $=1 \mathrm{~cm})$. (C) Micro-CT image of SA/Ge $/ C N C$ scaffold $(\mathrm{SA}: \mathrm{Ge}=2: 1, \mathrm{CNC}=0.5 \%)(\mathrm{diameter}=10$ $\mathrm{mm}$ ). (D) FTIR spectra of SA/Ge scaffold, CNC, and SA/Ge/CNC scaffold.

CNC showed characteristic absorption bands at around $2900 \mathrm{~cm}^{-1}, 3000 \mathrm{~cm}^{-1}$ and $1430 \mathrm{~cm}^{-1}$, which belong to $\mathrm{C}-\mathrm{H}$ stretching, $\mathrm{O}-\mathrm{H}$ stretching and $\mathrm{CH}_{2}$ scissoring band, respectively. ${ }^{42}$ A higher absorption from $3600 \mathrm{~cm}^{-1}$ to $3200 \mathrm{~cm}^{-1}$ appeared in the spectrum of $\mathrm{SA} / \mathrm{Ge} / \mathrm{CNC}$ scaffold, which suggested an increase of hydrogen bonds resulting from the interaction between SA/Ge backbone and CNC. ${ }^{36}$ The results of FTIR indicated the presence of CNC in the hybrid scaffold as wells as the interaction between them.

\subsection{Selection of the optimum ratio of $\mathrm{CNC}$ in $\mathrm{SA} / \mathrm{Ge} / \mathrm{CNC}$ scaffold}

CNC is a tough, biodegradable and renewable polymer which is primarily obtained from cellulose fibers. Owing to its distinctive properties, CNC was utilized in our study and acted as a reinforcing agent in polymer nanocomposites. To further explore the influence of CNC on the properties of scaffold and select its optimum concentration, cross-section morphology observation, water absorption ability, mechanical strength, and contact angle were systemically examined. The SEM images of SA/Ge/ CNC scaffolds were presented in Fig. 3A, which showed a porous and entangled network structure. The average pore size and size distributions were measured to study the effect of
CNC on the interconnectivity and homogeneity of scaffolds (Table 2). As the concentration of CNC enhanced from 0 to 1\%, the pore diameters shrank from $210 \pm 40 \mu \mathrm{m}$ to $120 \pm 16 \mu \mathrm{m}$. The pore sizes of $0.1 \%$ CNC group and $0.5 \%$ CNC group were $183 \pm 27 \mu \mathrm{m}$ and $157 \pm 31 \mu \mathrm{m}$, respectively. The pore sizes displayed a gradual decreasing trend with increasing CNC concentration. Unfortunately, some collapse appeared in the inner structure of SA/Ge/CNC scaffold with $1 \%$ CNC, which may attribute to the aggregation of $\mathrm{CNC}$ at such concentration. The results suggested that the addition of CNC could improve the pore numbers and diminish the pore sizes.

Fig. 3B displayed the water absorption properties of SA/Ge/ CNC scaffolds. The results showed that all the samples attained high swelling degree and the SA/Ge scaffold without CNC reached equilibrium swelling within a relatively shorter time. The swelling degree of control group (without CNC) was $627 \%$, while that of $1 \%$ CNC group significantly dropped to $207 \%$. The swelling degrees of $0.1 \%$ CNC group and $0.5 \%$ CNC group were $468 \%$ and $256 \%$, respectively. It can be observed from the figure that the incorporation of CNC significantly influenced the swelling behavior of scaffolds and increased concentrations of CNC resulted in the decline of swelling degree. The reason can be considered that the addition of CNC 

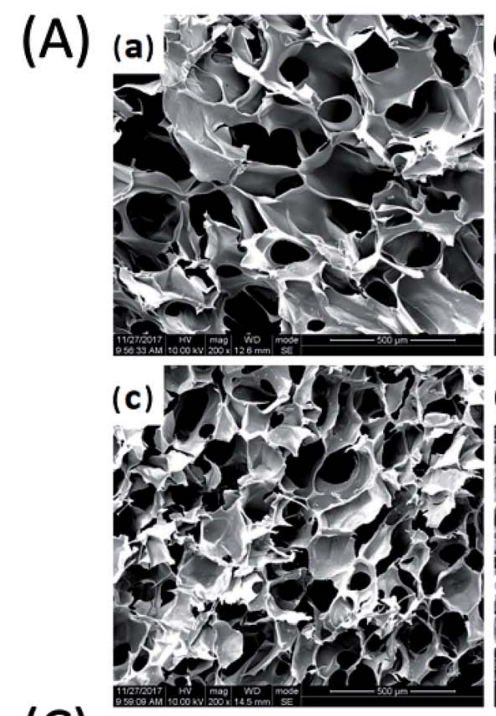

(C)

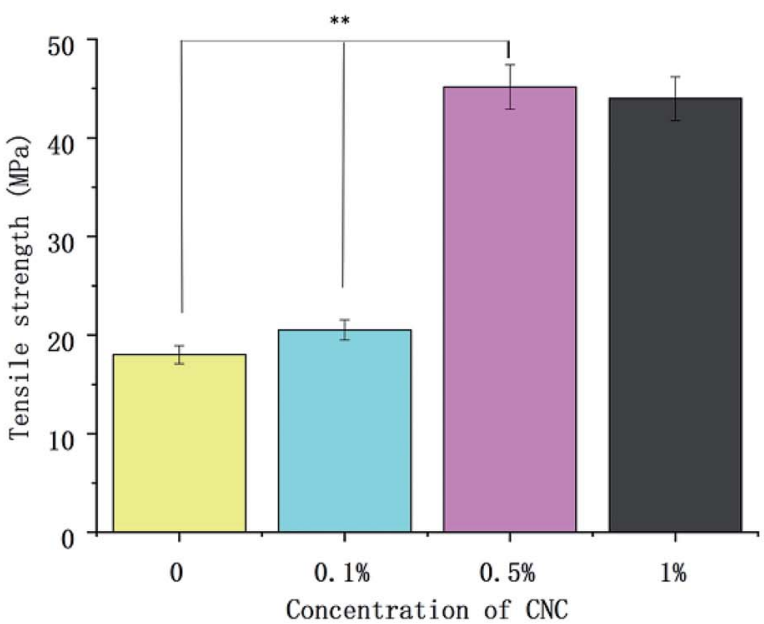

(E)

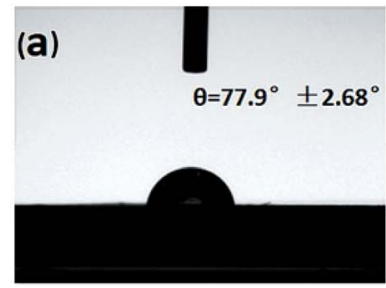

(b)
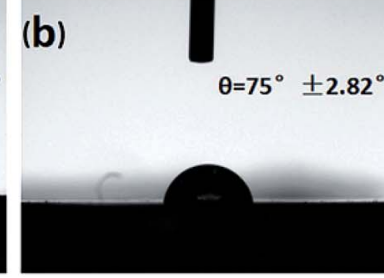

(B)
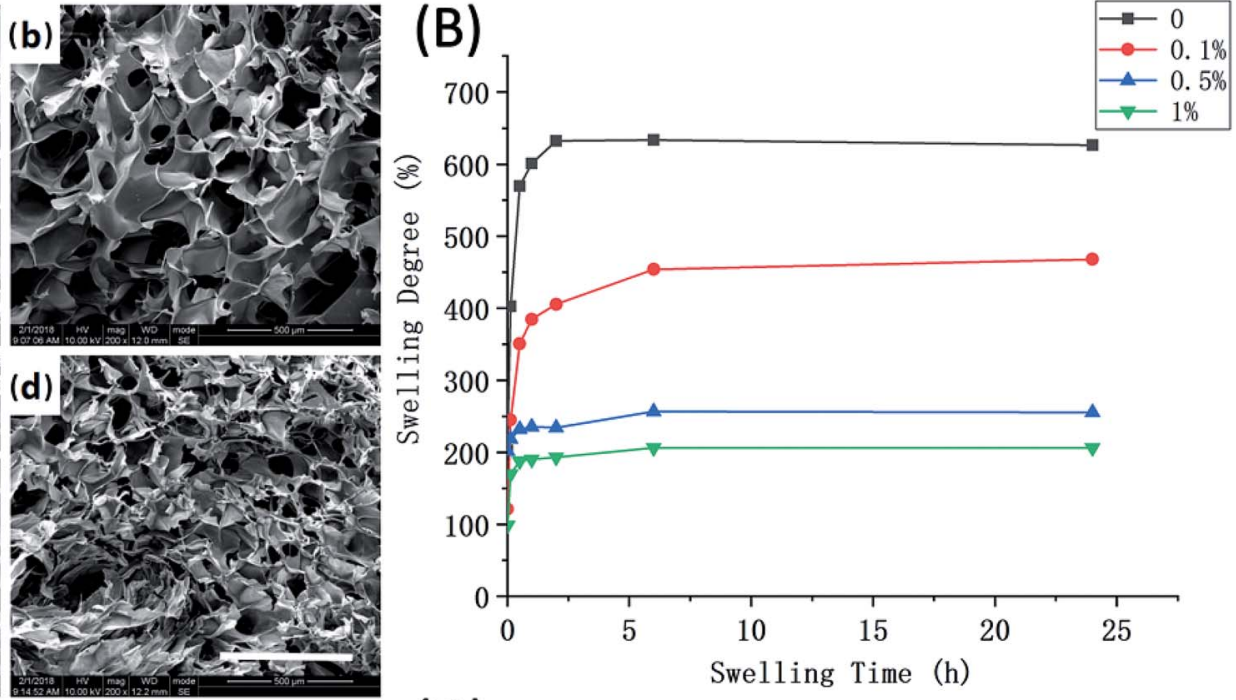

(D)

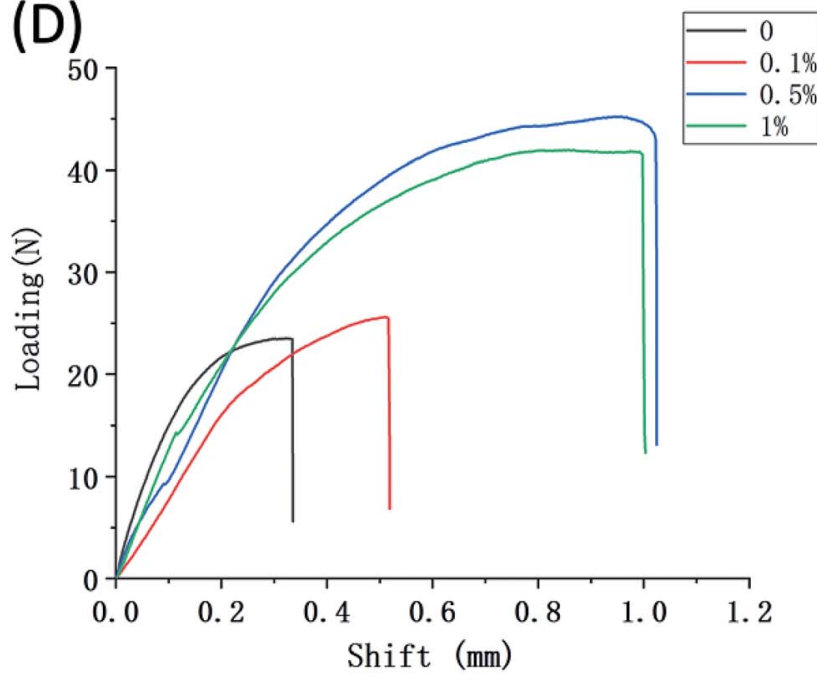

(c)

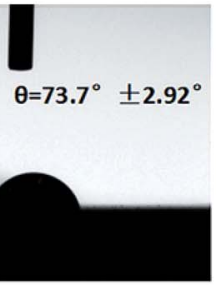

(d)

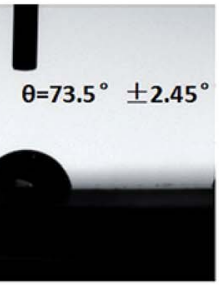

Fig. 3 Physical characteristics of SA/Ge/CNC (SA/Ge $=2: 1)$ scaffolds with different concentrations of CNC (A) SEM images of SA/Ge/CNC scaffolds: (a) without CNC, (b) $0.1 \%$ CNC, (c) $0.5 \%$ CNC, (d) $1 \%$ CNC. The bar corresponds to $500 \mu \mathrm{m}$. (B) Swelling degree, (C) maximum tensile strength at breaking points, (D) representative shift-loading curves, and (E) contact angles of SA/Ge/CNC scaffolds with different CNC concentration: (a) without CNC, (b) $0.1 \%$ CNC, (c) $0.5 \%$ CNC, (d) $1 \%$ CNC. ** denote significant difference at $p<0.01$ level.

renders the scaffold a denser and tighter network, thus less likely to absorb water.

The mechanical properties of $\mathrm{SA} / \mathrm{Ge} / \mathrm{CNC}$ scaffolds with different CNC contents were summarized in Fig. 3C and D. The results showed that SA/Ge scaffold without CNC had a tensile strength of 18.2 MPa, which enhanced to 20.5 MPa after the addition of $0.1 \%$ CNC. It's worth noting that the tensile strength dramatically raised to $45.1 \mathrm{MPa}$ after adding $0.5 \% \mathrm{CNC}$ into the scaffold. However, the tensile strength of $1 \%$ CNC group showed no much significant difference with the $0.5 \% \mathrm{CNC}$ group ( $p>0.05$ ). The results indicated that the mechanical strength of scaffolds showed a tendency to increase after the involvement of CNC. Due to the formation of hydrogen bonding, good interaction and compatibility of $\mathrm{CNC}$ with the composite components, CNC can provide significant enhancement in mechanical properties at low volume percentages. The increased amount of $\mathrm{CNC}$ in the $\mathrm{SA} / \mathrm{Ge} / \mathrm{CNC}$ scaffolds led to the formation of denser and more highly entangled network, thus resulting in the comparable stiffness improvement. 
Contact angle is an essential indicator to judge the hydrophobicity as well as hydrophilicity of materials. A decrease of water contact angle indicates the improvement of hydrophilicity. ${ }^{43}$ Previous studies have reported that hydrophilic biocomposites could promote the cell proliferation and the wettability is highly related to the barrier properties of materials. ${ }^{44}$ Here, the contact angles of different SA/Ge/CNC scaffolds were measured to study the impact of CNC on hydrophilicity. As shown in Fig. 3E, contact angles of all the samples were below $90^{\circ}$ and consistently at $\sim 75^{\circ}$ on average. The contact angles of control group, $0.1 \% \mathrm{CNC}$ group, $0.5 \% \mathrm{CNC}$ group and $1 \% \mathrm{CNC}$ group were $77.9^{\circ} \pm 2.68^{\circ}, 75^{\circ} \pm 2.82^{\circ}, 73.7^{\circ} \pm 2.92^{\circ}, 73.5^{\circ} \pm$ $2.45^{\circ}$, respectively. A slight downwards tendency of contact angles could be observed with the enhancement of CNC concentration. There was no significant difference between them. Therefore, CNC has no apparent effect on the hydrophilicity of scaffolds. On the other hand, the results confirmed the hydrophilicity nature of simple SA/Ge and SA/Ge/CNC scaffolds.

In consideration of the microstructure, swelling degree, tensile strength and contact angle characteristics mentioned above, the SA/ Ge/CNC scaffold containing $0.5 \%$ CNC exhibited superior properties and was selected to further explore its bio-applications for in vitro cytotoxicity assay and in vivo animal experiments.

\subsection{Cell study on SA/Ge/CNC scaffold}

An indispensable factor for tissue engineering materials is the capacity to support cell attachment, spreading and proliferation..$^{23}$ Non-cytotoxicity and biocompatibility of materials are crucial to further clinical applications. To assess the cytotoxicity of SA/Ge/CNC scaffold, 3T3 cells were incubated in $\mathrm{SA} / \mathrm{Ge} / \mathrm{CNC}$ scaffold leachate with different concentrations for $24 \mathrm{~h}$. The optical density of absorbance (O.D.) which reflects the cell viability was tested through a standard MTT method. As depicted in Fig. 4A, the experimental groups (concentrations of $\mathrm{SA} / \mathrm{Ge} / \mathrm{CNC}$ scaffold leachate $=0.78,1.56,3.13,6.25,12.5,25$, 50,75 and $100 \mathrm{mg} \mathrm{ml}^{-1}$ ) presented higher O.D. values than the control group (concentration of $\mathrm{SA} / \mathrm{Ge} / \mathrm{CNC}$ scaffold leachate $=$ $0 \mathrm{mg} \mathrm{ml} \mathrm{m}^{-1}$ ), suggesting that higher number of cells in the scaffold leachate of experimental groups than in the control group. The result indicates that the $\mathrm{SA} / \mathrm{Ge} / \mathrm{CNC}$ scaffold is nontoxic and possesses good cytocompatibility.

Subsequently, the morphology, attachment, and proliferation of 3T3 cells on scaffold were investigated. The SEM images of $3 \mathrm{~T} 3$ cells seeded on the SA/Ge/CNC scaffolds after 3 days were presented in Fig. 4B. It is observed that cells adhered and spread randomly on the SA/Ge/CNC scaffold. Besides, 3T3 cells presented a spindle-like shape and attached with each other, exhibiting an aggregated growth state. The safety of scaffolds is highly related to the good biocompatibility of biopolymers and nanoparticles. According to previous research, the RGD sequence (Arg-Gly-Asp) of gelatin can enhance cell adhesion, differentiation and proliferation..$^{22,45}$ Additionally, the similar chemical structure between cellulose and SA endows the prepared SA/Ge/CNC scaffolds good compatibility and

(A)

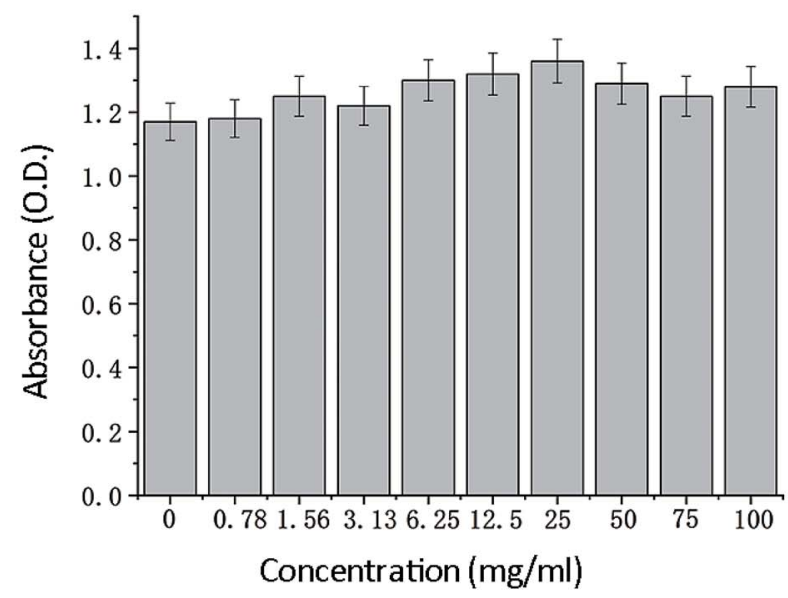

(B)
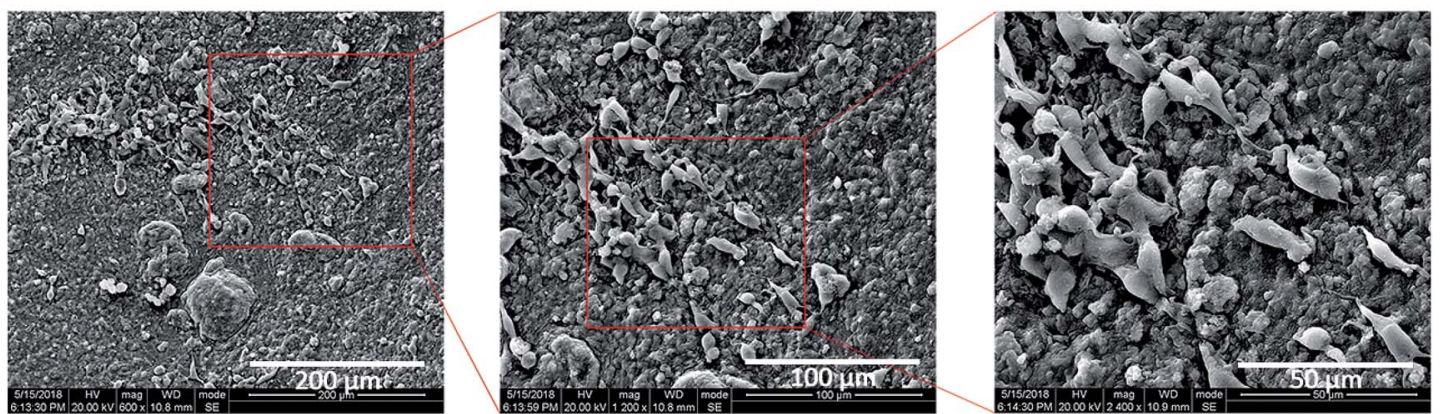

Fig. 4 Cell study on SA/Ge/CNC (SA/Ge $=2: 1, C N C=0.5 \%$ ) scaffold. (A) Cytotoxicity of 3T3 cells after 24 hour culture with scaffold leachate (MTT assay). (B) SEM images of $3 T 3$ cells cultured on SA/Ge/CNC scaffold for 3 days. 

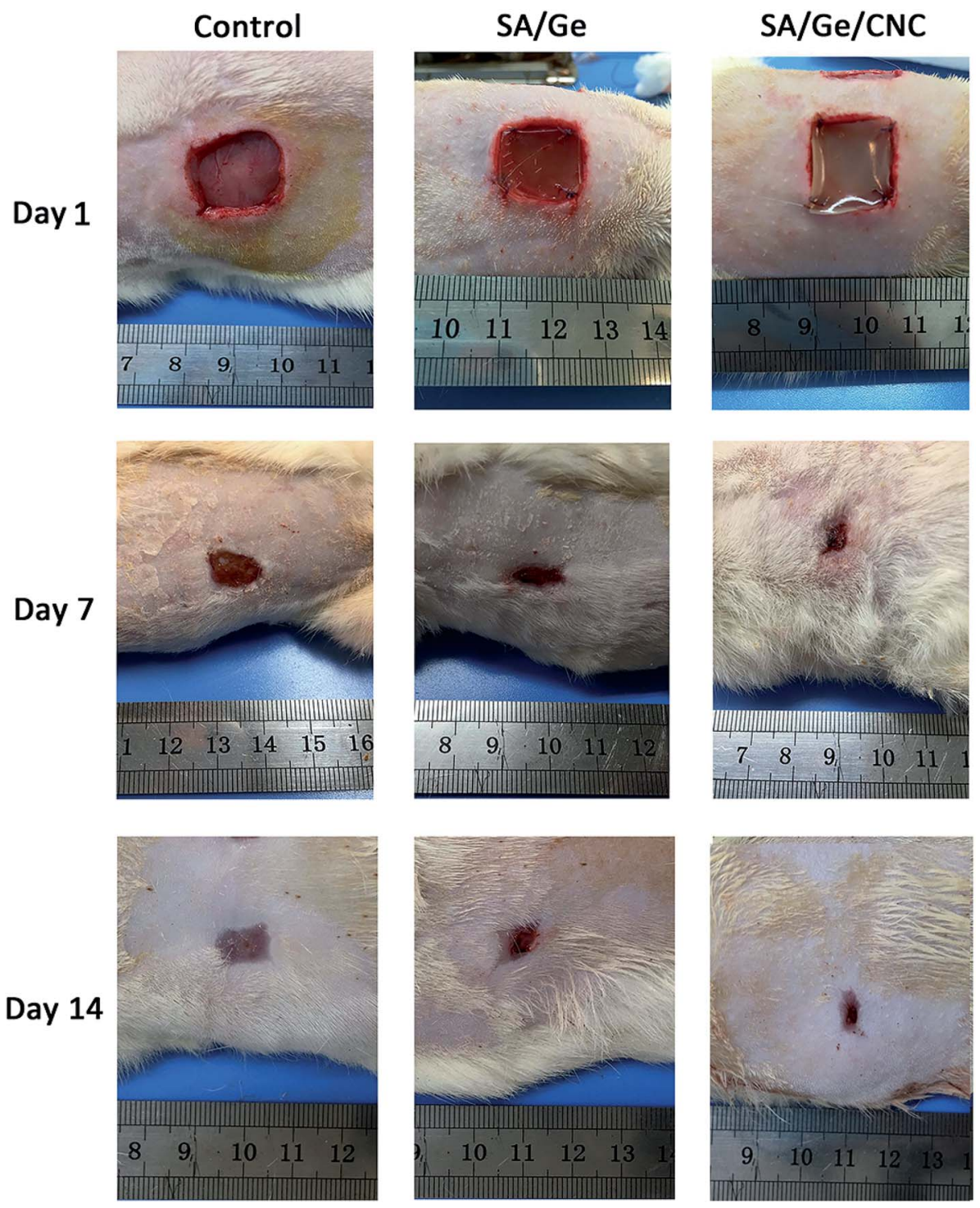

Fig. 5 Macroscopic observation of skin wounds in control, SA/Ge, and SA/Ge/CNC groups at 1 days, 7 days, and 14 days after surgery.

possibility of cross-linking reaction. Thus, the scaffolds form a better nanostructure for the growth and migration of cells. Briefly, the excellent biological properties of components might contribute to the successful adherence and good spread of 3T3 cells on the scaffold, indicating that $\mathrm{SA} / \mathrm{Ge} / \mathrm{CNC}$ scaffold is a potential candidate for wound regeneration applications.
(A)

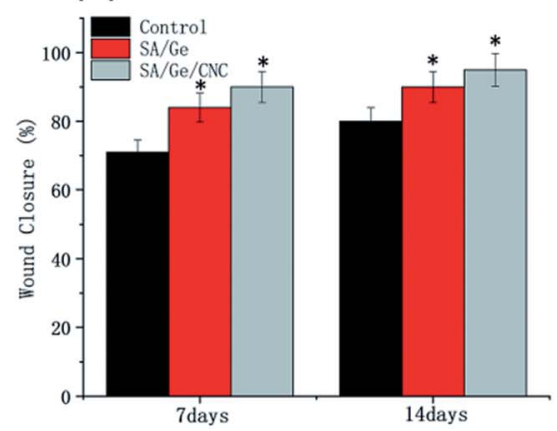

(B)

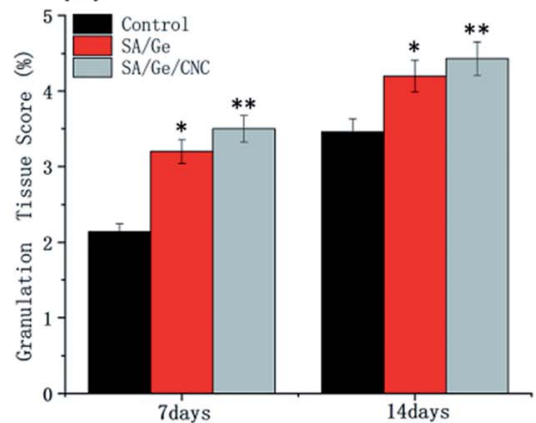

(C)

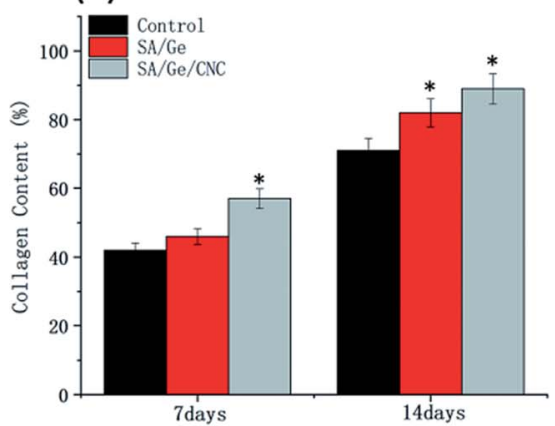

Fig. 6 (A) The wound closure, (B) granulation tissue score, (C) collagen content in control, SA/Ge, and SA/Ge/CNC groups at 7 days and 14 days after surgery. 

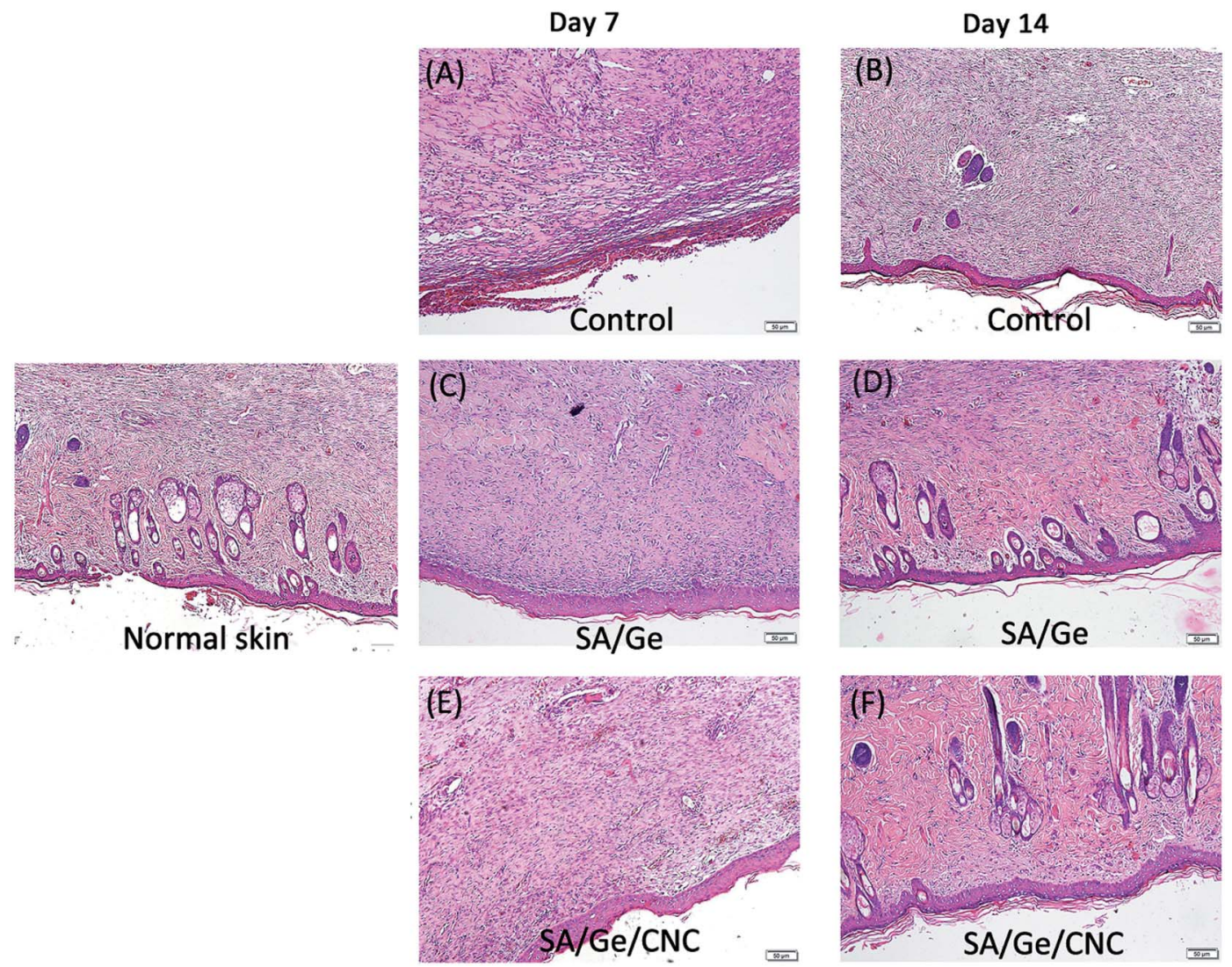

Fig. 7 H\&E staining images in control, SA/Ge, and SA/Ge/CNC groups at 7 days and 14 days after surgery. The bar corresponds to $50 \mu \mathrm{m}$.

\subsection{Skin regeneration in SD rats}

To further evaluate the wound healing performance of the SA/ Ge and SA/Ge/CNC scaffolds, two square defects $(1.5 \mathrm{~cm} \times 1.5$ $\mathrm{cm})$ were modeled on the left and right sides of each SD rat's dorsum. The scaffolds were covered on the right defects (experimental side) as dressings for the wounds, while the left defects were not treated with scaffolds and set as control. According to the scaffolds covered on the experimental sides, all the rats were randomly divided into two groups. Half of them were sutured with SA/Ge scaffolds (SA/Ge group), and the other half were sutured with $\mathrm{SA} / \mathrm{Ge} / \mathrm{CNC}$ scaffolds (SA/Ge/CNC group). Both kinds of scaffolds could be fixed on the four corners of defects with absorbable sutures and resisted the pull force (Fig. 5), which proved the remarkable mechanical strength of scaffolds.

On the 7 th and 14 th days after operation, digital photographs of wounds and the tissue sections were collected to quantitatively assess the area of wound closure, granulation tissue formation, and collagen deposition. Fig. 5 exhibits the macroscopic images of defects in the control, SA/Ge, and SA/Ge/CNC sides on day 1,7 , and 14 . During the wound healing progress, no apparent swelling or inflammatory reaction appeared on both sides of the two groups. As observed from photographs, the area of wounds reduced with time in all the groups. Compared to the control sides, the experimental sides of both groups exhibited faster wound healing rates and better skin recovery on day 7 and 14. It can be seen that the re-epithelialization of the experimental side in SA/Ge/CNC group were close to completion on day 14, whereas the same side in the SA/Ge group had not achieved the closure of the wound. At the same time, a large portion of the wound area in the control side was still not completely closed. The rate of re-epithelialization can be ranked in the order of $\mathrm{SA} / \mathrm{Ge} / \mathrm{CNC}>\mathrm{SA} / \mathrm{Ge}>$ control, revealing the better wound healing efficacy of $\mathrm{SA} / \mathrm{Ge} / \mathrm{CNC}$ scaffolds.

At different time points, the healing area of wounds were calculated according to a standard planimetric method. The wound closure percentages were expressed as the wound size reduction compared to the original area of defects (Fig. 6A). At day 14, the wound closure percentage of SA/Ge side and $\mathrm{SA} / \mathrm{Ge} / \mathrm{CNC}$ side reached $89 \%$ and $95 \%$, respectively. In contrast, the control side exhibited the lowest wound closure rate $(80 \%)$. The healing rate of SA/Ge/CNC side was higher than that of $\mathrm{SA} / \mathrm{Ge}$ side, which suggested that the $\mathrm{SA} / \mathrm{Ge} / \mathrm{CNC}$ scaffold has a better wound regeneration performance. Meanwhile, the granulation scores and the collage deposition scores were evaluated according to the optical microscope images of H\&E staining and Masson's trichrome staining (Fig. 6B and C). The granulation tissue formation and collage deposition play an essential role during the wound healing process and work as 
indicators to assess the regeneration of epithelium. The control side had the lowest granulation tissue accumulation and loosest collagen fibers on day 7 and 14. Fortunately, the SA/Ge/CNC side demonstrated a better recovery performance with 4.43 of granulation tissue formation score and $89 \%$ of collage deposition score after 14 days post-operation. The results indicated the positive function of SA/Ge/CNC scaffold on skin wound healing progress.

To further evaluate the wounding healing process, the reepithelialized skin along with normal skin was investigated histologically. The region of neo-epidermis, the formation of new epidermal annexes, and the deposition of collagen are considered as indicators of the early organization and remodeling of tissue. The histological analysis was performed on the basis of the observation of H\&E and Masson's trichrome staining results. In the micrographs of H\&E staining (Fig. 7), the thickness of the re-epithelialized skin and the infiltration of newly formed cells were clearly visible. At 7 days after treatment, we can find out that the $\mathrm{SA} / \mathrm{Ge}$ side and $\mathrm{SA} / \mathrm{Ge} / \mathrm{CNC}$ side possessed more continuous and compact epithelium tissue in the upper layer of wounds, whereas re-epithelialization in the control side was less pronounced. There was no obvious difference between the results of SA/Ge side and SA/Ge/CNC side on the 7th day post-injury. But on day 14, more microvessels proliferation and more skin appendage maturation such as hair follicles were observed in the $\mathrm{SA} / \mathrm{Ge} / \mathrm{CNC}$ side compared to the SA/Ge side. Such a difference suggested that wounds treated with $\mathrm{SA} / \mathrm{Ge} / \mathrm{CNC}$ scaffolds were at an accelerated rate of the healing process. The reformation and realignment of collagen are viewed as the major components of tissue remodeling phase during the wounding healing process. The improvement of the newly formed collagen contributed to the increase of tensile strength of tissue. ${ }^{\mathbf{4 6}}$ From the Masson's trichrome staining sections (Fig. 8), the extent and pattern of the collagen deposition can be compared according to the amount and density of the blue color. The light and dark blue represent the loosely interconnected and densely packed collagen bundles, respectively. At days 7 , the accumulation of collagen was loose and randomly organized in the control group. In contrast, the SA/ Ge group and SA/Ge/CNC group presented a higher amount of collagen fibers and appeared tightly packed. After 14 days, the difference got more apparent. Compared to the other two groups, images from the $\mathrm{SA} / \mathrm{Ge} / \mathrm{CNC}$ side demonstrated higher collagen density and more compact collagen alignment, where the presence of darker blue was noticed. The results of Masson's trichrome staining proved that $\mathrm{SA} / \mathrm{Ge} / \mathrm{CNC}$ scaffold could exert a positive effect on the dermal re-epithelialization and reconstruction, which were in agreement with the results of gross observation.

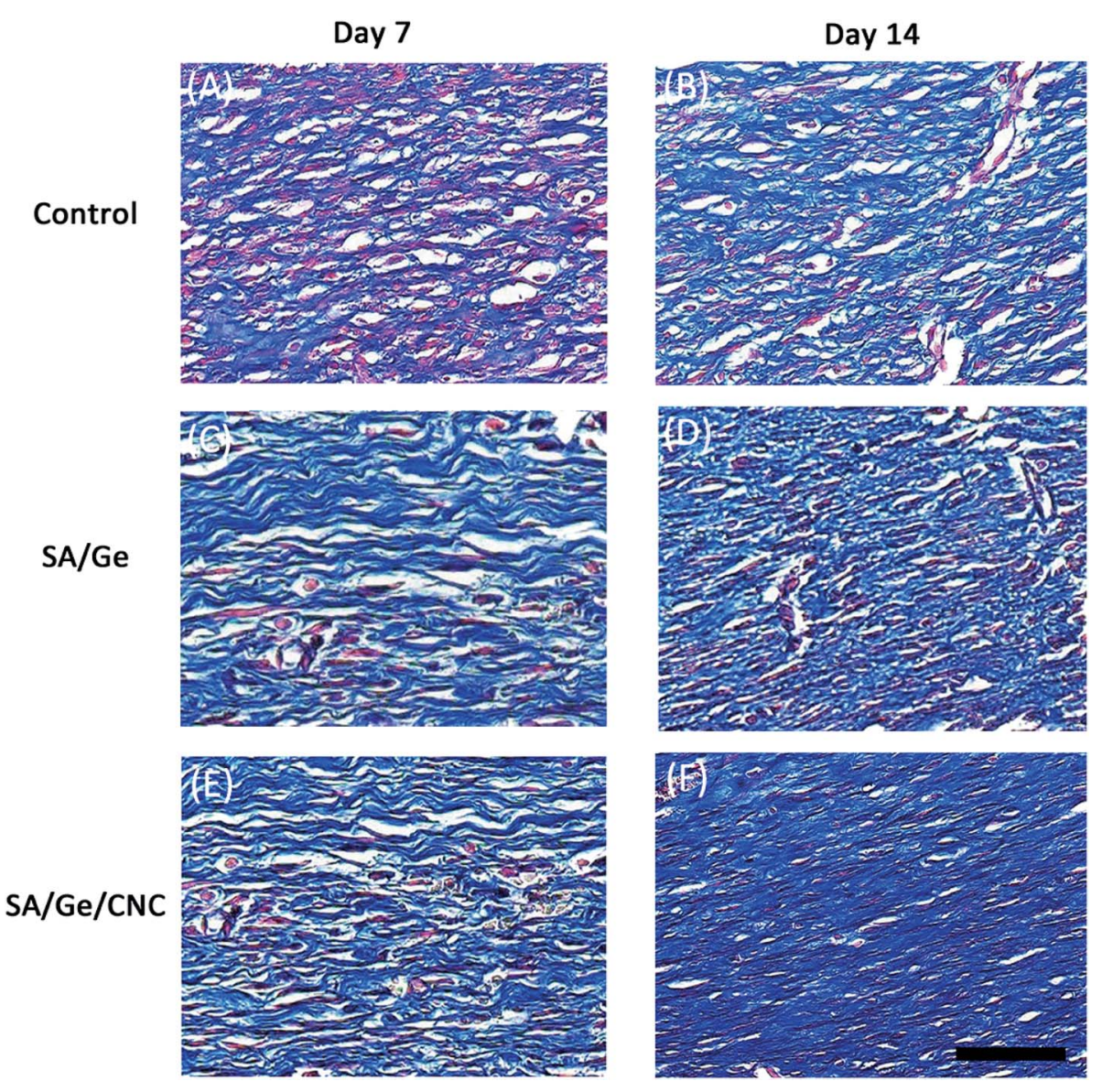

Fig. 8 Masson's trichrome staining images in control, SA/Ge, and SA/Ge/CNC groups at 7 and 14 days after surgery. The bar corresponds to 50 $\mu \mathrm{m}$. 


\section{Conclusion}

In summary, sodium alginate and gelatin scaffolds reinforced by CNC were prepared via a simple and cost-effective method. The optimum ratio of constituents was selected systemically. The microstructure and mechanical strength of SA/Ge scaffolds were significantly improved with the reinforcement of CNC. 3T3 cells can effectively attach and spread on the scaffold, which confirmed its good cyto-compatibility. In the in vivo skin regeneration experiment on $\mathrm{SD}$ rats, SA/Ge/CNC scaffolds demonstrated high efficiency to accelerate the recovery of wounds. Taken together, the SA/Ge/CNC scaffold with porosity, appropriate water absorption capacity, excellent mechanical property and biocompatibility is a desirable candidate for skin tissue engineering.

\section{Conflicts of Interest}

The authors declare no conflicts of interest.

\section{Acknowledgements}

The authors would like to thank the financial support from the National Natural Science Foundation of China (31600778) and Sichuan University Spark Project (2018SCUH0029). The authors would like to thank Dr Li Chen from Analytical \& Testing Center Sichuan University for her help with micro-CT scanning and analysis. All the animal procedures were performed in accordance with the Guidelines for Care and Use of Laboratory Animals of Sichuan University and approved by the Animal Ethics committee of State Key Laboratory of Oral Diseases at Sichuan University.

\section{References}

1 M. P. Sousa, A. I. Neto, T. R. Correia, S. P. Miguel, M. Matsusaki, I. J. Correia and J. F. Mano, Biomater. Sci., 2018, 6, 1962-1975.

2 C. Qi, L. Xu, Y. Deng, G. Wang, Z. Wang and L. Wang, Biomater. Sci., 2018, 6, 2859-2870.

3 J. Liao, Y. Jia, B. Wang, K. Shi and Z. Qian, ACS Biomater. Sci. Eng., 2018, 4, 1029-1036.

4 M. Rezvanian, M. Amin and S. F. Ng, Carbohydr. Polym., 2016, 137, 295-304.

5 B. K. Boekema, M. Vlig, L. Olde Damink, E. Middelkoop, L. Eummelen, A. V. Buhren and M. M. Ulrich, J. Mater. Sci.: Mater. Med., 2014, 25, 423-433.

6 R. Quan, X. Zheng, S. Xu, L. Zhang and D. Yang, Stem Cell Res. Ther., 2014, 5, 118.

7 M. Mohiti-Asli, S. Saha, S. V. Murphy, H. Gracz, B. Pourdeyhimi, A. Atala and E. G. Loboa, J. Biomed. Mater. Res., Part B, 2017, 105, 327-339.

8 T. Agarwala, R. Narayana, S. Majia, S. Beheraa, S. Kulanthaivelb, T. Kumar Maitia, I. Banerjeeb, K. Palb and S. Giri, Int. J. Biol. Macromol., 2016, 93, 1499-1506.

9 M. Khamrai, S. L. Banerjee and P. P. Kundu, Carbohydr. Polym., 2017, 174, 580-590.
10 P. Yuan, X. Qiu, R. Jin, Y. Bai, S. Liu and X. Chen, Biomater. Sci., 2018, 6, 820-826.

11 Q. Zhu, M. Jiang, Q. Liu, S. Yan, L. Feng, Y. Lan, G. Shan, W. Xue and R. Guo, Biomater. Sci., 2018, 6, 2472-2486.

12 J. Venkatesan, I. Bhatnagar, P. Manivasagan, K. H. Kang and S. K. Kim, Int. J. Biol. Macromol., 2015, 72, 269-281.

13 Q. Huang, L. Hao, J. Xie, T. Gong, J. Liao and Y. Lin, ACS Appl. Mater. Interfaces, 2015, 7, 20893-20901.

14 D. J. Overstreet, D. Dutta, S. E. Stabenfeldt and B. L. Vernon, J. Polym. Sci., Part B: Polym. Phys., 2012, 50, 881-903.

15 J. Boateng, R. Burgos-Amador, O. Okeke and H. Pawar, Int. J. Biol. Macromol., 2015, 79, 63-71.

16 A. Saarai, V. Kasparkova, T. Sedlacek and P. Saha, J. Mech. Behav. Biomed. Mater., 2013, 18, 152-166.

17 L. Yuan, Y. Wu, Q. S. Gu, H. El-Hamshary, M. El-Newehy and X. Mo, Int. J. Biol. Macromol., 2017, 96, 569-577.

18 V. Gopalakannan and N. Viswanathan, Int. J. Biol. Macromol., 2016, 83, 450-459.

19 E.-S. Chan, S.-L. Wong, P.-P. Lee, J.-S. Lee, T. B. Ti, Z. Zhang, D. Poncelet, P. Ravindra, S.-H. Phan and Z.-H. Yim, Carbohydr. Polym., 2011, 83, 225-232.

20 D. Singh, A. Tripathi, S. Zo, D. Singh and S. S. Han, Colloids Surf., B, 2014, 116, 502-509.

21 N. El Miri, K. Abdelouahdi, A. Barakat, M. Zahouily, A. Fihri, A. Solhy and M. El Achaby, Carbohydr. Polym., 2015, 129, 156-167.

22 B. Balakrishnan and R. Banerjee, Chem. Rev., 2011, 111, 4453-4474.

23 X. Hu, L. Lu, C. Xu and X. Li, Int. J. Biol. Macromol., 2015, 72, 403-409.

24 K. Song, Y. Yang, S. Li, M. Wu, Y. Wu, M. Lim and T. Liu, Mater. Sci. Eng., C, 2014, 40, 197-203.

25 K. Y. Lee and D. J. Mooney, Prog. Polym. Sci., 2012, 37, 106126.

26 N. Alemdar, Carbohydr. Polym., 2016, 151, 1019-1026.

27 J. George and S. N. Sabapathi, Nanotechnol., Sci. Appl., 2015, $8,45-54$.

28 H. Dong, K. E. Strawhecker, J. F. Snyder, J. A. Orlicki, R. S. Reiner and A. W. Rudie, Carbohydr. Polym., 2012, 87, 2488-2495.

29 K. Madhusudana Rao, A. Kumar and S. S. Han, Int. J. Biol. Macromol., 2017, 101, 165-171.

30 J. R. Yu, J. Navarro, J. C. Coburn, B. Mahadik, J. Molnar, J. H. t. Holmes, A. J. Nam and J. P. Fisher, Adv. Healthcare Mater., 2019, 8, e1801471.

31 H. Sun, K. Feng, J. Hu, S. Soker, A. Atala and P. X. Ma, Biomaterials, 2010, 31, 1133-1139.

32 J. Yan, Y. Miao, H. Tan, T. Zhou, Z. Ling, Y. Chen, X. Xing and X. Hu, Mater. Sci. Eng., C, 2016, 63, 274-284.

33 Y. Wang, Z. Wang, K. Wu, J. Wu, G. Meng, Z. Liu and X. Guo, Carbohydr. Polym., 2017, 168, 112-120.

34 L. Tu, Y. He, H. Yang, Z. Wu and L. Yi, J. Biomater. Sci., Polym. Ed., 2015, 26, 735-749.

35 N. Naseri, B. Deepa, A. P. Mathew, K. Oksman and L. Girandon, Biomacromolecules, 2016, 17, 3714-3723.

36 C. Miao and W. Y. Hamad, Cellulose, 2013, 20, 2221-2262. 
37 T. Huq, S. Salmieri, A. Khan, R. A. Khan, C. Le Tien, B. Riedl, C. Fraschini, J. Bouchard, J. Uribe-Calderon, M. R. Kamal and M. Lacroix, Carbohydr. Polym., 2012, 90, 1757-1763.

38 N. Lin, C. Bruzzese and A. Dufresne, ACS Appl. Mater. Interfaces, 2012, 4, 4948-4959.

39 K. Song, L. Li, R. Li, M. Lim, P. Liu and T. Liu, Appl. Biochem. Biotechnol., 2014, 173, 838-850.

40 C. Yang, H. Frei, F. M. Rossi and H. M. Burt, J. Tissue Eng. Regener. Med., 2009, 3, 601-614.

41 A. Z. Aroguz, K. Baysal, Z. Adiguzel and B. M. Baysal, Appl. Biochem. Biotechnol., 2014, 173, 433-448.
42 C. Kumar, M. U. H. Joardder, T. W. Farrell, G. J. Millar and M. A. Karim, Drying Technol., 2015, 34, 962-973.

43 N. Pal, P. Dubey, P. Gopinath and K. Pal, Int. J. Biol. Macromol., 2017, 95, 94-105.

44 G. Siqueira, J. Bras and A. Dufresne, Biomacromolecules, 2009, 10, 425-432.

45 M. Angelozzi, M. Miotto, L. Penolazzi, S. Mazzitelli, T. Keane, S. F. Badylak, R. Piva and C. Nastruzzi, Mater. Sci. Eng., C, 2015, 56, 141-153.

46 S. Udhayakumar, K. G. Shankar, S. Sowndarya and C. Rose, Biomater. Sci., 2017, 5, 1868-1883. 